\title{
Hepatoprotección antioxidante de la cáscara y semilla de Vitis vinifera $L$. (uva)
}

\author{
Antioxidant liver protection of Vitis vinifera L. (grape) skin and seed
}

\author{
Miguel Sandoval ${ }^{1}$, Karen Lazarte ${ }^{1}$, Inés Arnao ${ }^{1}$ \\ ${ }^{1}$ Centro de Investigación de Bioquímica y Nutrición Alberto Guzmán Barrón. Facultad de Medcina, Universidad Nacional Mayor de San Marcos. Lima, Perú.
}

\section{Resumen}

Objetivo: Determinar la capacidad hepatoprotectora antioxidante, inducida por las semillas y cáscaras de la uva Vitis vinifera L., en animales de experimentación con agresión alcohólica, mediante la prueba del TBARS (sustancias reactivas al ácido tiobarbitúrico). Diseño: Estudio experimental. Institución: Centro de Investigación de Bioquímica y Nutrición Alber to Guzmán Barrón, Facultad de Medicina, Universidad Nacional Mayor de San Marcos, Lima, Perú. Material biológico: Ratones albinos, machos, adultos y cáscaras y semillas de Vitis vinifera L. (uva). Métodos: Las cáscaras y semillas de uva fueron del valle de Cañete; se las separó manualmente, se las exprimió en gasa y fueron desecadas con aire circulante, a $40^{\circ} \mathrm{C}$, por 24 horas; luego, fueron trituradas, y la mezcla de cáscaras y semillas (cas-sem) fue administrada ad libitum en la dieta. Se utilizó 104 ratones albinos machos adultos, separados en grupos, a los que se administró: (A) cas-sem al 20\%; (B) alcohol al 5\%; (C) cas-sem con alcohol; (D) silimarina $50 \mathrm{mg} / 100 \mathrm{~g}$ de alimento; (E) silimarina con alcohol; y, (F) grupo control. Bajo anestesia con éter y mediante laparotomía, se extrajo los hígados, fueron pesados y analizados por lipoperoxidación, mediante TBARS, y se evaluó la hepatomegalia, por peso a las 24, 48 y 72 horas, y a los 4, 5 y 7 días de tratamiento. Principales medidas de resultados: Lipoperoxidación hepática y hepatomegalia. Resultados: La hepatomegalia se presentó desde las 24 horas (36,68\% de incremento de masa hepática), en el grupo alcohol, y fue menor en el grupo cas-sem. La prueba TBARS fue mayor en el grupo alcohol (63,91 a 67,07 $\mathrm{nmol} / \mathrm{g}$-tejido) y fue menor en el grupo cas-sem (40,85 a 47,46 nmol/g-tejido); en el grupo cas-sem con alcohol, fue 43 a 63 nmol/g-tejido y la protección se observó hasta el quinto día (44 nmol/g-tejido). Conclusiones: El cas-sem administrado en la dieta, al $20 \%$ en peso, protege al tejido hepático, hasta el quinto día de injuria constante con alcohol al $5 \%$, por vía digestiva.

Palabras clave: Hepatopatías; TBARs; Vitis; antioxidantes; peroxidación de lípido.

\begin{abstract}
Objective: To determine by TBARS test (substances reactive to thiobarbituric acid) the antioxidant and liver protection effect induced by Vitts vinifera L. grape skin and seeds in animals of experimentation with alcoholic aggression. Design: Experimental study. Setting: Alberto Guzman Barron Biochemistry and Nutrition Research Center, Faculty of Medicine, Universidad Nacional Mayor de San Marcos, Lima, Peru. Biologic material: Albino male adult mice and Vitis vinifera L. (grape) skin and seeds. Methods: Grape skin and seeds were brought from Cañete Valley; they were separated manually, squeezed in gauze and dissecated with flowing air at $40^{\circ} \mathrm{C}$ for 24 hours; then they were grinded and the skin and seeds mixture (skin-seeds) were administered ad libitum in the diet. We used 104 albino male adult mice, separated in groups, that received: (A) skin-seeds at $20 \%$; (B) alcohol at 5\%; (C) skin-seeds with alcohol; (D) silimarina $50 \mathrm{mg} / 100 \mathrm{~g}$ of food; (E) silimarina with alcohol; and, (F) control group. Under ether anesthesia and by laparotomy, liver was removed, weighted and analyzed for lipoperoxidation by TBARS, and hepatomegaly was determined by weight at 24, 48 and 72 hours, and at 4, 5 and 7 days of treatment. Main outcome measures: Liver lipoperoxidation and hepatomegaly. Results: Hepatomegaly was observed at 24 hours (36,68\% liver mass increment) in the alcohol group and was less in the skins-seeds group. TBARS test was higher in the alcohol group (63,91 to $67,07 \mathrm{nmol} / \mathrm{g}$-tissue) and was less in the skin-seeds group ( 40,85 to $47,46 \mathrm{nmol} / \mathrm{g}$-tissue); in the skin-seeds with alcohol group it was 43 to $63 \mathrm{nmol} / \mathrm{g}$-tissue and protection was seen up to the fifth day (44 nmol/g-tissue). Conclusions: Skin-seeds administered orally in the diet at $20 \%$ of weight protected liver tissue up to the fifth day of constant injury with $5 \%$ alcohol.
\end{abstract}

Key words: Liver diseases, TBARs; Vitis; antioxidants; lipid peroxidation.

\section{INTRODUCCIÓN}

En la producción de vino, uno de los primeros residuos, después de haber realizado la extracción del jugo de la uva, es el conjunto de cáscaras, fibra y semillas del fruto usado, el cual posteriormente es desechado sin uso potencial ${ }^{(1,2)}$. En la actualidad, cada vez más se observa una relación directa de las enfermedades degenerativas, metabólicas y de larga data -como diabetes, aterosclerosis, hipertensión arterial, enfermedades cardiovasculares, entre otras-, con la producción de radicales libres y agentes prooxidantes ${ }^{(3-5)}$.

Una de las enfermedades sociales, presente desde la antigüedad, es el alcoholismo. El alcohol es una molécula que produce daño a nivel celular, tanto ella misma como los productos de su metabolismo. Uno de los principales órganos que afecta es el hígado ${ }^{(6-8)}$. El alcohol es una molécula con gran capacidad de incrementar el estrés oxidativo celular ${ }^{(4)}$.

La apoptosis celular es uno de los principales tipos de muerte celular programada. La apoptosis es un fenómeno biológico fundamental, permanente, dinámico e interactivo. Existen mecanismos pro y anti apoptóticos, regulados genéticamente, que actúan de forma activa (pues consumen energía) y equilibrada. La apoptosis está directamente asociada al incremento del estrés oxidativo ${ }^{(9,10)}$. La cáscara y semilla de la uva, así como el vino, tienen entre sus componentes a polifenoles, vitaminas $\mathrm{C}$ y $\mathrm{E}$, flavonoides, entre otros, todos ellos con capacidad antioxidante y que sugieren protección tisular frente al estrés oxidativo ${ }^{(11-13)}$. Por ello, nos propusimos observar y determinar la capacidad hepatoprotectora antioxidante, inducida por las semillas y cáscaras de la Vitis vinifera L. (uva), en polvo (cas-sem), en animales de experimentación con agresión alcohólica, mediante la prueba del TBARS (sustancias reactivas al ácido tiobarbiturico).

Las cáscaras y semillas son un desecho aprovechable de la uva que se obtiene del primer paso del proceso de obtención del vino. Entre sus componentes, existen compuestos antioxidantes que pueden servir, utilizando a estas cáscaras y semi- 
llas desecadas y trituradas (cas-sem) como materia prima base para el preparado de alimentos semi-procesados, como mermeladas, harina, fibra dietaria, entre otros. Los componentes antioxidantes de la uva y presentes en el vino, así como en otras frutas, verduras y alimentos vegetales, hacen tener a la mano alimentos que favorecen el cuidado y preservación de la salud ${ }^{(6,7)}$.

La asociación conocida del estrés oxidativo a enfermedades metabólicas -como diabetes mellitus-, degenerativas -como el cáncer-y otras -como las enfermedades cardiovasculares- estimulan la investigación de compuestos, fármacos y alimentos con capacidad antioxidante, a partir de diversos productos naturales, tales como la uva y sus derivados ${ }^{(9,11,13)}$.

El consumo social de alcohol y las consecuencias que genera su consumo, sobre todo en el hígado, es otro de los factores que fundamentan este estudio, toda vez que la investigación se realizará en el tejido hepático, analizando los daños que produce la ingesta de alcohol en animales de experimentación -midiendo el estrés oxidativo mediante la prueba de TBARS- y observando la protección con la ingesta de cáscaras y semillas de uva en otro grupo.

La protección del organismo frente al estrés oxidativo es natural, pero se encuentra relacionada a las moléculas que se ingiere en la dieta y las cáscaras y semillas de la uva; al tener entre sus componentes sustancias antioxidantes, pueden ser una alternativa de protección natural.

La 'paradoja francesa' constituyó un interesante punto de partida, al mostrar cómo en Francia existe un riesgo bajo de padecer enfermedades cardiovasculares en la población, a pesar del elevado consumo de grasas saturadas de origen animal. Renaud y col. ${ }^{(14)}$ descubrieron que la dieta de los franceses difiere de la de otros países desarrollados por el consumo de vino tinto. Los estudios epidemiológicos de Hertog y col. ${ }^{(15)}$, Hein y col. ${ }^{(16)}$ y McElduff y col. ${ }^{(17)}$ relacionan el consumo moderado y regular de vino con una disminución del riesgo de padecer enfermedades cardiovasculares, crónicas y degenerativas, realizados a raíz de la 'paradoja francesa'.
Las uvas y vinos contienen una gran variedad de compuestos fenólicos, de muy diversas estructuras químicas y pesos moleculares, cuya cantidad total y la proporción en que aparecen dependen de una serie de factores, como la variedad de la uva, el área de producción, la climatología, las técnicas agrícolas, los métodos de vinificación, el procedimiento de prensado de la uva y el tiempo de fermentación del mosto con la piel y las semillas ${ }^{(18-20)}$.

Los compuestos fenólicos de la uva se localizan en las partes sólidas: cáscara, semilla y tejido vascular. En la pulpa, destaca la presencia de ácidos fenólicos y sus derivados ${ }^{(21)}$. Los flavonoles y antocianos se encuentran localizados en las células de la cáscara de la uva, siendo estos últimos responsables del color rojo de los vinos tintos. Las procianidinas y flavanoles se localizan en las semillas de las uvas. En los vinos blancos, los fenoles mayoritarios son aquellos procedentes de la pulpa, mientras que en los tintos, la maceración alcohólica de los hollejos y las semillas permite la liberación y solubilización de los flavonoides $(13,22,23)$.

Las especies reactivas de oxígeno (ROS) son especies químicas radicales y no radicales que, debido a su inestabilidad, se comportan como agentes oxidantes. Las ROS pueden tener en nuestro organismo un origen endógeno relacionado con el metabolismo del oxígeno y con distintas reacciones de defensa de nuestro sistema inmunológico ${ }^{(24)}$. También, pueden provenir de fuentes externas: el tabaco, la contaminación del aire, la radiación ultravioleta y la de alta energía, el ozono o ciertos medicamentos. El organismo dispone de mecanismos de defensa antioxidante frente a las especies reactivas de oxígeno, que comprenden sistemas enzimáticos y no enzimáticos ${ }^{(25)}$.

Sies ${ }^{(24)}$ y Halliwell ${ }^{(25)}$ señalan que ciertas enzimas -como superóxido dismutasa, catalasa, glutatión peroxidasa o glutatión reductasa-, neutralizan las especies reactivas. Los sistemas defensivos no enzimáticos abarcan una serie de compuestos antioxidantes, como albúmina, ceruloplasmina, transferrina, glutatión, bilirrubina, ácido úrico, ubiquinona o melatonina. En ciertas situaciones ex- tremas, estas defensas no son suficientes y las especies reactivas producen daño oxidativo, tanto en biomoléculas como en componentes celulares ${ }^{(26)}$.

El daño oxidativo se relaciona con el origen y desarrollo de ciertas enfermedades multifactoriales de carácter crónico, como la oxidación de las LDL y la enfermedad cardiovascular, así lo refiere Berliner (27); también, está relacionado el daño oxidativo al $\mathrm{ADN}^{(21)}$ y la oxidación de las proteínas de las lentes oculares y la alteración de la visión, como lo refieren Dean y col. ${ }^{(28)}$ y Taylor A ${ }^{(29)}$.

Los estudios de Taylor ${ }^{(29)}$ y Gerster ${ }^{(30)}$ señalan que la ingesta de alimentos ricos en sustancias antioxidantes -como vitaminas $\mathrm{C}$ y E, carotenoides o compuestos fenólicos- previenen o disminuyen el desarrollo de estas enfermedades, señalando que la dieta aumenta la defensa antioxidante del organismo, evitando el daño oxidativo.

Los compuestos fenólicos son sustancias orgánicas ampliamente distribuidas en el reino vegetal. Se sintetizan como metabolitos secundarios, con funciones de defensa, y son en gran medida responsables de las propiedades del color, la astringencia y el flavor (sabor y aroma) de los vegetales. Se encuentran en las verduras, frutas y en productos derivados, como el vino o la cerveza. Todos tienen en su estructura uno o más anillos aromáticos, con al menos un sustituyente hidroxi, como lo describe Bravo L ${ }^{(31)}$. Su estructura química es propicia para secuestrar radicales libres, debido a la facilidad con la que el átomo de hidrógeno -desde el grupo hidroxilo aromático- puede ser donado a la especie radical, y a la estabilidad de la estructura quinona resultante, que soporta un electrón desapareado. La actividad antioxidante de los polifenoles depende del número y la localización de los grupos hidroxilo que contiene en su estructura. Además de su actividad antioxidante, se ha atribuido las siguientes propiedades biológicas a los compuestos fenólicos: Laughton ${ }^{(32)}$ indica que estos compuestos inhiben la agregación plaquetaria; Andriambeloson y col. (2) y Delmas ${ }^{(33)}$ indican su función como agentes vasodilatadores, antiinflamatorios y anticancerígenos. 
Los radicales libres (RL) son moléculas que se derivan del oxígeno, están en continua formación en las células del organismo y en pequeñas cantidades no producen efectos tóxicos. En situación normal, la producción de radicales libres es constante en una concentración determinada y son neutralizados por las defensas antioxidantes, que pueden ser sustancias propias del organismo (las enzimas antioxidantes) o pueden ser sustancias presentes en algunos alimentos, como la vitamina $\mathrm{C}$, vitamina $\mathrm{E}$, el beta caroteno, flavonoides, entre otros. ${ }^{(34,35)}$.

Cuando se produce un desequilibrio, ya sea por mayor producción de RL o menor acción de los antioxidantes, el organismo se encuentra sometido al denominado estrés oxidativo, que genera efectos tóxicos y patologías, fundamentalmente enfermedades aterioescleróticas (enfermedades coronarias, accidentes cerebro vasculares, obstrucción vascular de los miembros inferiores, aneurismas arteriales) (36). También, se producen daños en los ácidos nucleicos, lesiones o mutaciones celulares, que derivan en la aparición de determinados tumores. En ese sentido, los antioxidantes ingeridos a través de la dieta serían útiles para la prevención de estas patologías. Los vinos rojos contienen sustancias de la familia de los flavonoides, compuestos químicos antioxidantes que se obtiene, entre otras fuentes naturales, de la cáscara de la uva negra. Además de los flavonoides, en el vino tinto existen otros componentes -como el alcohol- que no son beneficiosos para la salud. En cuanto al aporte de nutrientes del vino (vitaminas, minerales, proteínas), no es considerable, y su aporte calórico se puede obtener de otros alimentos más saludables ${ }^{(3,23,33)}$.

Los compuestos polifenólicos de la uva se encuentran en la piel, especialmente en las células epidérmicas, y en las semillas. Su concentración es baja en la pulpa. Esto explica por qué el vino blanco, que no se hace con la semilla ni la piel, presenta niveles bajos de polifenoles. En este sentido, el más rico en estas sustancias es el vino tinto cabernet sauvignon ${ }^{(9,18)}$. La cantidad de polifenoles en la uva depende principalmente de la variedad de la vid, del clima, del terreno y de las prácticas de cultivo. La capacidad antioxidante del vino se encuentra directamente relacionada con su contenido en polifenoles. La contribución de cada compuesto en particular depende no solo de su concentración y de su calidad antioxidante, sino que también de su interacción con otros componentes. Los compuestos interactúan entre sí, lo que puede producir efectos sinérgicos o inhibitorios.

Estudios epidemiológicos y experimentales han mostrado una correlación entre el consumo moderado de vino y la prevención de los accidentes cardiovasculares. En este proceso, el resveratrol tendría, por sus propiedades antitrombóticas, un papel inhibidor de la agregación plaquetaria o de antioxidante de lipoproteínas y pared vascular ${ }^{(5,36)}$. En la actualidad, las investigaciones se centran en la acción del resveratrol sobre la expresión de genes diana implicados en la proliferación celular, a dos niveles: estudio del mecanismo responsable del efecto antiproliferativo del resveratrol en función de la dosis utilizada en hepatomas y en otras líneas de células cancerosas humanas (en particular colorrectales), e investigaciones dirigidas a determinar si los procesos de apoptosis juegan un papel en el proceso $y$, en caso afirmativo, estudiar la sensibilidad frente a los distintos tipos celulares. Los resultados preliminares muestran una fuerte inhibición por resveratrol de la proliferación de una línea celular de cáncer colorrectal asociada a un proceso de apoptosis, según Delmas y col ${ }^{(33)}$.

Es de resaltar la actual importancia de la toxicidad que conllevan los radicales libres durante los procesos biológicos, donde una de las consecuencias del estrés oxidativo es la peroxidación lipídica, cuya prevención es esencial en todos los organismos aerobios, ya que los productos derivados de este proceso pueden interactuar con el ADN y son potencialmente mutágenos. Los epóxidos formados pueden reaccionar espontáneamente con centros nucleofílicos en la célula o unirse a los ácidos nucleicos (ADN y ARN). Esta reacción puede dar lugar a citotoxicidad, alergia, mutagénesis o carcinogénesis, dependiendo de las propiedades del epóxido en cuestión. En los organismos aerobios existe una gran variedad de sistemas de defensa antioxidante, tanto enzimáticos como no enzimáticos, que se coordinan cooperativamente y protegen al organismo de los riesgos que conlleva el estrés oxidativo. Entre ellos, destacan las actividades enzimáticas superóxido dismutasa (SOD), glutatión peroxidasa (GPX) y catalasa (CAT), glutatión (GSH), además del ácido ascórbico (vitamina C), alfa-tocoferol (vitamina E), beta-caroteno, vitamina $\mathrm{A}$, flavonoides y ácidos fenólicos ${ }^{(21)}$.

\section{MÉTODOS}

Se realizó un estudio de tipo experimental, utilizándose 104 ratones albinos de laboratorio (Mus musculus) cepa Balb/c/ $\mathrm{CNPB}$, machos, adultos, de un peso entre 25 a $30 \mathrm{~g}$, a quienes se les administró dieta ad libitum, y se distribuyó aleatoriamente 16 ratones en los siguientes 6 grupos: (1) grupo control, con alimento balanceado; (2) grupo cas-sem, con alimento y extracto de semillas y cáscaras de la Vitis vinifera L. (uva) en polvo (cas-sem), al 20\%; (3) grupo alcohol, con alimento balanceado y alcohol al $5 \%$ en el agua del bebedero; (4) grupo cas-sem con alcohol, con alimento balanceado con cas-sem al $20 \%$ y alcohol al $5 \%$ en el agua del bebedero; (5) grupo silimarina, con alimento balanceado y silimarina $50 \mathrm{mg} / 100 \mathrm{~g}$ del alimento; y, (6) grupo silimarina con alcohol, con alimento balanceado y silimarina $50 \mathrm{mg} / 100 \mathrm{~g}$ del alimento y alcohol al 5\% en el agua del bebedero. En cada uno de los grupos, la evaluación del tejido hepático para medir el nivel de estrés oxidativo se realizó a las 24 horas, 48 horas, 72 horas, 5 y 7 días.

La silimarina es un principio activo de la planta Silybum marianum, popularmente conocida como cardo mariano, cuyos ingredientes activos son flavonoides, como silibina, silidianina y silicristina; tienen acción hepatoprotectora y regenerativa: los mecanismos de acción son su actividad antioxidante, por su capacidad para contrarrestar la acción de los radicales libres que se forman por acción de las toxinas y que dañan las membranas celulares (peroxidación lipídica), la Inhibición competitiva -modificando la membrana celular externa de los hepatocitos, lo cual forma un complejo que impide el ingreso de toxinas al interior de las células del hígado y la estimulación me- 
tabólica de las células hepáticas-, activa la biosíntesis del ARN de los ribosomas, estimulando la formación de proteínas, por lo que nos sirve como un compuesto de de comparación.

Para las pruebas de laboratorio, se empleó equipos e instrumentos del laboratorio III-2 del Centro de Investigación de Bioquímica y Nutrición Alberto Guzmán Barrón, de la Facultad de Medicina de la Universidad Nacional Mayor de San Marcos. Se usó para cada grupo de animales, jaulas, bebederos y comederos suficientes y alimento balanceado para ratones.

Se utilizó uva negra proveniente del valle de Cañete, de la especie Vitis vinifera L., madura. Previo lavado de la uva, se separó la cáscara y las semillas, descartando el contenido del fruto; estas fueron colocadas en gasa y exprimidas manualmente, para retirar restos de jugo del fruto, colocadas sobre una fuente con papel secante, la cual se llevó a estufa de flujo de aire caliente a temperatura de $40^{\circ} \mathrm{C}$ por 24 horas. Se obtuvo así las semillas y cáscaras secas y posteriormente se las trituró en molino de alimentos, obteniéndose un polvo fino de cáscaras y semillas al cual denominamos cas-sem.

El alimento balanceado de los animales fue obtenido del Instituto Nacional de Salud-Lima; se trituró en molino de alimentos, obteniendo un polvo fino; este se mezcló con el cas-sem, tomando una parte de cas-sem con cuatro partes de alimento balanceado, constituyéndose así una dieta con $20 \%$ de cas-sem. El alcohol fue preparado al $5 \%$ en agua envasada, comercial.

Posteriormente, los animales fueron anestesiados con vapores de éter dietílico y pesados; luego, se realizó una laparoscopia, se localizó el ligamento redondo mayor del hígado, se pinzó y se extrajo el hígado, cortando los ligamentos y adherencias secundarias. El hígado fue lavado en solución fisiológica de cloruro de sodio al 0,9 g\% y se le secó en papel adsorbente. Posteriormente, se pesó todo el órgano en una balanza analítica. Se encontró la relación porcentual del peso del hígado/ peso del animal. El tejido hepático fue colocado en un tubo graduado y se agregó buffer fosfato $50 \mathrm{mmol} / \mathrm{L}$, a pH 7,4, hasta completar $10 \mathrm{~mL}$ y se llevó al homogenizador de teflón, a velocidad constante de $300 \mathrm{rpm}$, durante 30 segundos, para que el tejido se homogenizara. En el homogenizado de hígado, se determinó el estrés oxidativo, mediante la evaluación de la lipoperoxidación o peroxidación lipídica por la prueba de las especies reactivas al ácido tiobaritúrico, por método espectrofotométrico a $535 \mathrm{~nm}$.

Los estadísticos descriptivos usados fueron la media o promedio aritmético, la desviación de estándar y la variación porcentual. Se usó la prueba t de student de la diferencia para el análisis de comparación entre los grupos, para un nivel de significancia de $p<0,05$ en una gráfica de dos colas, comparando el grupo cas-sem y grupo alcohol con el grupo control, el grupo cas-sem alcohol con el grupo cassem y el grupo silimarina alcohol con grupo silimarina. Para el análisis respectivo, se utilizó el paquete estadístico SPSS 13,0 .

\section{RESULTADOS}

El consumo de cas-sem en el alimento y los volúmenes de alcohol ingerido en los diferentes grupos no tuvieron diferencias significativas $(p>0,1)$.

La relación del peso del hígado/peso del animal, con 24 horas de tratamiento de los diferentes grupos, fue mayor en el grupo que ingirió alcohol $(5,79 \%)$, con un incremento de $+36,68 \%$ del tamaño del hígado, denotando hepatomegalia, de manera significativa $(p=0,0019)$. El menor incremento de la relación porcentual peso del hígado/peso del animal fue en el grupo cas-sem $(4,24 \%)$, que representa hepatomegalia, correspondiente a un aumento de 13,61\%. La comparación de los grupos silimarina y silimarina alcohol, no mostró diferencia significativa ( $p=$ $0,6735)$ (tabla 1).

A las 48 horas de tratamiento, el grupo que observó mayor relación del peso del hígado/peso del animal fue el grupo alcohol $(5,80 \%)$, que denotó un crecimiento hepático de 23,78\% (hepatomegalia), con diferencia significativa $(p=0,0397)$. El grupo cas-sem alcohol no tuvo diferencia $(p=0,0944)$. La comparación de los grupos silimarina y silimarina alcohol no mostró diferencia significativa $(p=0,749)$ (tabla 1).

La relación del peso del hígado/peso del animal en los grupos con 72 horas de tratamiento fue mayor en el grupo que ingirió alcohol $(7,68 \%)$, con un incremento de $+58,25 \%$ del tamaño del hígado, denotando hepatomegalia, de manera significativa $(p=0,0021)$. El menor incremento de la relación porcentual peso del hígado/peso del animal fue en el grupo cas-sem $(5,28 \%)$, que representa hepatomegalia, correspondiente a un aumento de $8,88 \%$, variación no significativa $(p=0,1564)$. La comparación de los grupos silimarina y silimarina alcohol fue de $6,12 \%$ de hepatomegalia, cifra sin diferencia significativa $(p=0,4733)$. (tabla 1).

A los 7 días de tratamiento, la relación porcentual del peso hepático/peso del animal fue mayor en el grupo que consumió alcohol (7,13\%), observándose una hepatomegalia correspondiente a $44,32 \%$, con diferencia significativa $(p=0,0061)$; mientras tanto, en el grupo cas-sem alcohol no hubo variación significativa $(p=0,1907)$. La hepatomegalia del grupo silimiarina alcohol, en comparación al grupo silimarina, ocurrió en $10,32 \%$, incremento sin diferencia significativa $(p=0,2083)$ (tabla 1$)$.

Al observar la variación de la relación porcentual peso del hígado/peso del animal en el tiempo de tratamiento, existe en el grupo alcohol un constante incremento, siendo el mayor a las 72 horas; además, los valores que denotan hepatomegalia siempre fueron mayores en el grupo alcohol que en los otros tratamientos. El tratamiento con cas-sem y cas-sem alcohol tuvieron elevación de la relación porcentual peso del hígado/ peso del animal, pero no significativa (figura 1).

La silimarina, medicamento que nos sirvió de comparación, mostró su efecto protector tras observar la relación peso hígado/peso del animal \% (hepatomegalia), siendo sus valores siempre menores que los demás grupos y no existiendo diferencia significativa entre el grupo con silimarina y el de silimarina alcohol, demostrando así la protección de la silimarina, como se muestra en la figura 2. 
Tabla 1. Relación porcentual del peso del hígado/peso del animal y porcentaje de hepatomegalia de Mus muscullus con tratamientos, a las 24,48 y 72 horas y a los 7 días.

\begin{tabular}{|c|c|c|c|c|c|c|c|c|}
\hline Grupo & $\mathrm{n}$ & $\begin{array}{c}\mathrm{X} \\
\text { hig/W\% }\end{array}$ & $\mathrm{DE}$ & EE & $\begin{array}{c}\text { Hepatomegalia } \\
\%\end{array}$ & $\begin{array}{c}\mathrm{t} \\
\text { student }\end{array}$ & $p$ & Obs \\
\hline \multicolumn{9}{|l|}{ A las 24 horas } \\
\hline Control & 4 & 3,72 & 0,375 & 0,175 & --- & --- & --- & --- \\
\hline Cas-sem & 4 & 4,24 & 0,291 & 0,136 & 13,61 & 2,15 & 0,0731 & No sig \\
\hline Alcohol & 4 & 5,79 & 0,692 & 0,323 & 36,68 & 5,26 & 0,0019 & Sig \\
\hline Cas-sem + alcohol & 4 & 5,08 & 0,552 & 0,257 & 19,99 & 2,73 & 0,0342 & Sig \\
\hline Silimarina & 4 & 5,16 & 0,909 & 0,424 & --- & --- & --- & --- \\
\hline Silimarina + alcohol & 4 & 5,48 & 1,137 & 0,530 & 6,22 & 0,44 & 0,6735 & No Sig \\
\hline \multicolumn{9}{|l|}{ A las 48 horas } \\
\hline Cas-sem & 4 & 4,68 & 0,541 & 0,252 & --- & --- & --- & --- \\
\hline Alcohol & 4 & 5,80 & 0,668 & 0,311 & 23,78 & 2,61 & 0,0397 & Sig \\
\hline Cas-sem + alcohol & 4 & 5,24 & 0,146 & 0,068 & 11,82 & 1,89 & 0,0944 & No sig \\
\hline Silimarina & 4 & 5,60 & 0,485 & 0,226 & --- & --- & --- & --- \\
\hline Silimarina + alcohol & 4 & 5,90 & 0.637 & 0,297 & 5,34 & 0,75 & 0,749 & No Sig \\
\hline \multicolumn{9}{|l|}{ A las 72 horas } \\
\hline Cas-sem & 4 & 4,85 & 0,215 & 0,100 & --- & --- & --- & --- \\
\hline Alcohol & 4 & 7,68 & 1,083 & 0,505 & 58,25 & 5,144 & 0,0021 & Sig \\
\hline Cas-sem + alcohol & 4 & 5,28 & 0,494 & 0,230 & 8,88 & 1,6197 & 0,1564 & No sig \\
\hline Silimarina & 4 & 5,93 & 0,187 & 0,087 & --- & --- & --- & --- \\
\hline Silimarina + alcohol & 4 & 6,29 & 0,932 & 0,435 & 6,12 & 0,7649 & 0,4733 & No sig \\
\hline \multicolumn{9}{|l|}{ A los 7 días } \\
\hline Cas-sem & 4 & 4,49 & 0,421 & 0,196 & --- & --- & --- & --- \\
\hline Alcohol & 4 & 7,13 & 0,974 & 0,454 & 44,32 & 4,1314 & 0,0061 & Sig \\
\hline Cas-sem + alcohol & 4 & 5,42 & 0,491 & 0,229 & 9,65 & 1,4749 & 0,1907 & No sig \\
\hline Silimarina & 4 & 5,59 & 0,673 & 0,314 & --- & --- & --- & --- \\
\hline Silimarina + alcohol & 4 & 6,17 & 0,465 & 0,217 & 10,32 & 1,4096 & 0,2083 & No sig \\
\hline
\end{tabular}

$\mathrm{X}$ hig/W\%: promedio $(\mathrm{X})$ de la relación porcentual del peso del hígado entre el peso del animal.

La lipoperoxidación hepática (TBARS) basal observada en el grupo control fue de 55,52+6,08 nmol/g; después de 24 horas de tratamiento con cas-sem, la lipoperoxidación hepática fue

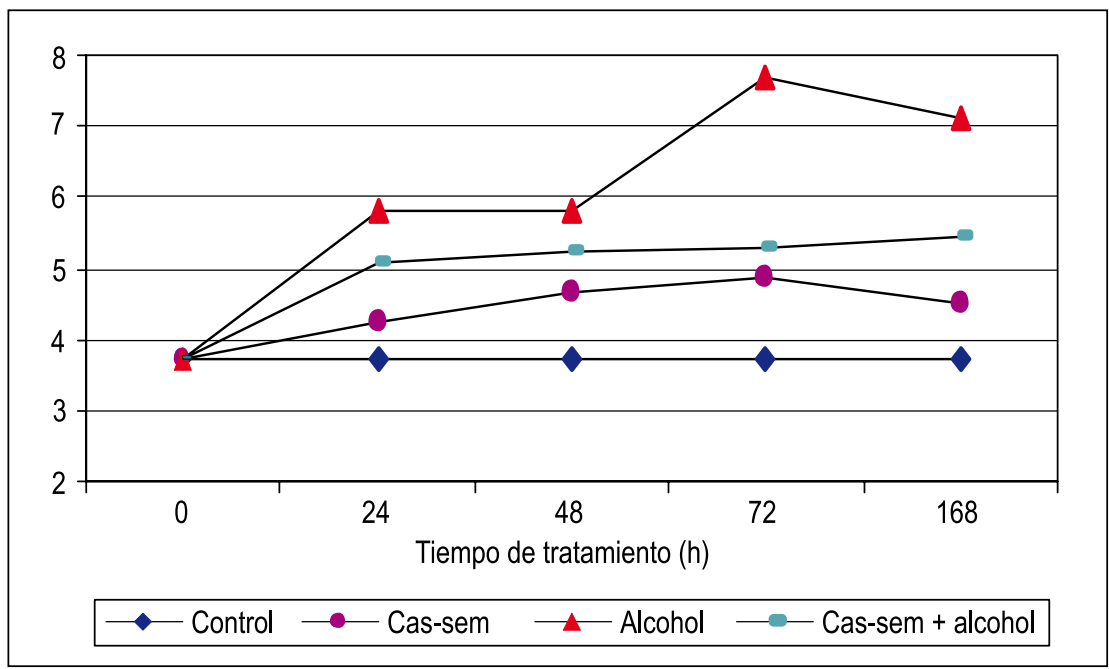

Figura 1. Variación de la relación porcentual del peso del hígado/peso del animal de Mus musculus, según tiempo y tratamiento.
$47,46+6,72 \mathrm{nmol} / \mathrm{g}$. En el grupo alcohol, fue $63,91 \mathrm{nmol} / \mathrm{L}$ y, en el grupo cas-sem alcohol, fue 43,71 nmol/g, sin diferencia significativa. Los grupos tratados con silimarina con y sin alcohol presentaron

fue $45,54+4,21 \mathrm{nmol} / \mathrm{g}$, mientras que se apreció un incremento en los grupos alcohol $(67,07+8,15 \mathrm{nmol} / \mathrm{g})$ y cas-sem alcohol $(47,30+4,60 \mathrm{nmol} / \mathrm{g})$. El incremento de lipoperoxidación en el grupo alcohol fue significativamente alto $(p=$ 0,003 ) y la disminución de la lipoperoxidación en el grupo tratado con cas-sem fue significativamente menor $(p=0,035)$. La peroxidación lipídica en los grupos de silimarina y silimarina alcohol fueron menores y entre ellos no hubo diferencia significativa $(p=0,479)$ (tabla 2$)$.

La lipoperoxidación a las 72 horas de tratamiento fue significativamente menor en el grupo que consumió cassem $(41,55+7,08 \mathrm{nmol} / \mathrm{g} ; p=0,024)$. La comparación de la lipoperoxidación entre los grupos silimarina y silimarina alcohol 
no muestra diferencia significativa $(p=$ 0,091) (tabla 2).

A los 7 días de tratamiento, el valor de la lipoperoxidación en el grupo cas-sem siguió reduciéndose $(40,85+4,31 \mathrm{nmol} / \mathrm{g})$, con diferencia significativa $(p=0,007)$, y la de los grupos alcohol y cas-sem alcohol se incrementaron a $61,91+10,36 \mathrm{nmol} / \mathrm{g}$ y $63,10+4,20 \mathrm{nmol} / \mathrm{g}$, respectivamente ambos de manera significativa. Los valores en los grupos silimarina y silimarina alcohol fueron menores, y entre ellos fue mayor el grupo que consumió alcohol (29,11 y $35,74 \mathrm{nmol} / \mathrm{g}$, respectivamente), sin diferencia significativa entre ellos $(p=, 232)$ (tabla 2$)$.

Al observar el cambio de la lipoperoxidación según el tiempo de tratamiento, claramente se aprecia la agresión del alcohol hasta las 48 horas, aunque desciende a las 72, luego vuelve a elevarse; en cambio, en el grupo cas-sem alcohol se encuentra protegido hasta las 72 horas, luego se eleva la lipoperoxidación a los 7 días, como se observa en la figura 3 .

En la comparación de los valores del daño oxidativo, expresado en porcentaje de daño o protección hepática, es evidente el daño producido por el alcohol, siendo mayor a las 48 horas, pero con tendencia elevada; la protección que brindó el cas-sem a la agresión del alcohol (grupo cas-sem alcohol) fue hasta las 48 horas; menor a las 72 horas, recuperó la protección a los 7 días (168 horas). En los animales que consumieron cas-sem y no tuvieron la agresión del alcohol, la protección fue cada día mayor, con tendencia a estabilizarse (figura 4).

\section{DISCUSIÓN}

En la actualidad, se conoce el efecto de los antioxidantes en la salud del hombre. Históricamente, se le atribuyó este rol a la vitamina E, posteriormente se extendió a otras moléculas, pero fueron los estudios epidemiológicos los que dieron luces del efecto antioxidante. Varias de estas investigaciones demostraron que el consumo de vino era saludable y que reducía significativamente la morbilidad y mortalidad por enfermedades cardiovasculares. Entre estas demostraciones se tiene el consumo de frutas, verduras y consumo moderado

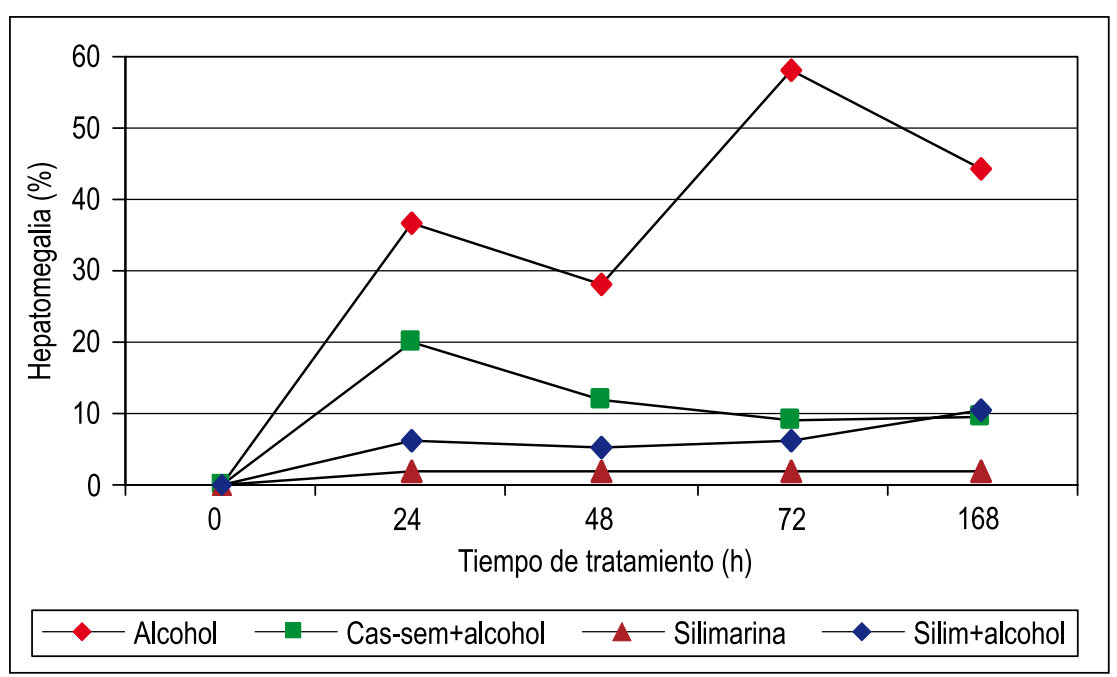

Figura 2. Variación de la relación peso hígado/peso animal de Mus musculus, según tiempo y tratamiento.

de vino, demostrando una reducción incluso de las placas ateromatosas. Estudios posteriores demuestran que el consumo de alimentos que contienen compuestos fenólicos -como frutas frescas, verduras, aceite de oliva, uvas y vino dependiendo su maduración ${ }^{(36)}$-, disminuye el riesgo de sufrir de enfermedades cardiovasculares ${ }^{(37)}$.

Estudios de investigación científica revelan que la mortalidad celular y la enfermedad se deben a desórdenes metabólicos en la célula, principalmente por el incremento del estrés oxidativo. El organismo posee ciertas defensas antioxidantes, basadas en sistemas enzimáticos y substratos intracelulares; entre estos se tiene a la superóxido dismutasa, catalasa, glutatión peroxidasa, vitaminas $\mathrm{C}$ y E, uratos, glutatión, entre otros.

El alcoholismo es una enfermedad en la que se ve incrementado el estrés oxidativo, que origina una serie de distorsiones funcionales hepáticas. Uno de los signos de alteración de la función del tejido hepático es el aumento del tamaño celular, que conlleva a un incremento en el volumen y peso del hígado, que se conoce como hepatomegalia. Es por esta razón que, una de las evaluaciones iniciales y cuantitativas que realizamos fue medir el peso del hígado en los animales de los diferentes grupos tratados.

La relación porcentual peso del hígado/peso del animal mostró rápidamente un incremento en aquellos casos en los que se administró alcohol, a las primeras 24 horas de manera significativa, $36,68 \%$ más respecto al grupo control $(p=0,0019)$. En los animales que recibieron cas-sem, se observó incremento de $13,61 \%$, pero no significativo $(p=$ $0,731)$. Y, en los que recibieron cas-sem alcohol, la hepatomegalia fue de 19,99\%, elevación con significancia ( $p=0,0342)$, pero menor que la del grupo de animales que ingirió alcohol solo, observándose ya una protección del cas-sem frente a la injuria del alcohol, tal como se aprecia en el tabla 1.

A las 48 horas de tratamiento, se observó hepatomegalia en el grupo de animales que ingirió alcohol, aumentando de $13,69 \%$ a $23,78 \%$ la relación del peso del hígado/peso del animal; y, respecto al grupo que consumió cas-sem con alcohol, el porcentaje de 19,99\% (a las 24 horas) se redujo a $11,82 \%$ a las 48 horas, observándose así el efecto hepatoprotector del cas-sem sobre la injuria del alcohol, esto en función a que no existe diferencia sigificativa con el grupo que consumió solo cas-sem ( $p=$ 0,0944) (tabla 1).

El crecimiento del hígado o hepatomegalia es un signo común que antecede principalmente a la cirrosis hepática, causada por alcoholismo o como secuela de hepatitis A o B, pero que también puede indicar la presencia de algún tipo 
Tabla 2. Lipoperoxidación (TBARs) en tejido hepático de Mus muscullus con tratamientos, a las 24,48 y 72 horas y a los 7 días.

\begin{tabular}{|c|c|c|c|c|c|c|c|c|}
\hline Grupo & $n$ & $\begin{array}{c}\mathrm{X} \\
\text { TBARS }\end{array}$ & $\mathrm{DE}$ & EE & $\begin{array}{c}\text { Daño } \\
\%\end{array}$ & $\begin{array}{c}\mathrm{t} \\
\text { student }\end{array}$ & $p$ & Obs. \\
\hline \multicolumn{9}{|l|}{ A las 24 horas } \\
\hline Control & 4 & 55,52 & 6,08 & 2,83 & 0 & --- & --- & --- \\
\hline Cas-sem & 4 & 47,46 & 6,72 & 3,13 & $-14,51$ & 1,77 & 0,125 & No sig \\
\hline Alcohol & 4 & 63,91 & 7,51 & 3,50 & $+15,11$ & 1,74 & 0,130 & No sig \\
\hline Cas-sem + alcohol & 4 & 43,71 & 8,87 & 4,13 & $-7,90$ & 2,19 & 0,07 & No sig \\
\hline Silimarina & 4 & 37,28 & 9,55 & 4,45 & $-21,44$ & --- & --- & --- \\
\hline Silimarina + alcohol & 4 & 39,86 & 3,19 & 1,48 & $-16,01$ & 0,51 & 0,626 & No sig \\
\hline \multicolumn{9}{|l|}{ A las 48 horas } \\
\hline Control & 4 & 55,52 & 6,08 & 2,83 & 0 & --- & --- & --- \\
\hline Cas-sem & 4 & 45,51 & 4,21 & 1,96 & $-18,03$ & 2,70 & 0,035 & Sig \\
\hline Alcohol & 4 & 67,07 & 8,15 & 3,80 & $+20,80$ & 4,70 & 0,003 & Sig \\
\hline Cas-sem + alcohol & 4 & 47,30 & 4,60 & 2,14 & $+3,93$ & 0,57 & 0,586 & No sig \\
\hline Silimarina & 4 & 33,05 & 7,40 & 3,45 & $-27,37$ & -- & --- & --- \\
\hline Silimarina + alcohol & 4 & 36,59 & 5,80 & 2,70 & $-19,60$ & 0,75 & 0,479 & No sig \\
\hline \multicolumn{9}{|l|}{ A las 72 horas } \\
\hline Control & 4 & 55,52 & 6,08 & 2,83 & 0 & --- & --- & --- \\
\hline Cas-sem & 4 & 41,55 & 7,08 & 3,30 & $-25,16$ & 2,99 & 0,024 & Sig \\
\hline Alcohol & 4 & 52,40 & 10,42 & 4,85 & $+5,61$ & 1,71 & 0,135 & No sig \\
\hline Cas-sem + alcohol & 4 & 52,74 & 5,31 & 2,47 & $+26,93$ & 2,52 & 0,044 & Sig \\
\hline Silimarina & 4 & 32,38 & 3,48 & 1,62 & $-22,06$ & -- & -- & --- \\
\hline Silimarina + alcohol & 4 & 40,92 & 7,76 & 3,61 & $-1,51$ & 2,00 & 0,091 & No sig \\
\hline \multicolumn{9}{|l|}{ A los 7 días } \\
\hline Control & 4 & 55,52 & 6,08 & 2,83 & 0 & --- & --- & --- \\
\hline Cas-sem & 4 & 40,85 & 4,31 & 2,01 & $-26,42$ & 3,93 & 0,007 & Sig \\
\hline Alcohol & 4 & 61,91 & 10,36 & 4,83 & $+11,51$ & 3,75 & 0,0095 & Sig \\
\hline Cas-sem + alcohol & 4 & 63,10 & 4,20 & 1,95 & $+13,65$ & 7,39 & 0,0003 & Sig \\
\hline Silimarina & 4 & 29,11 & 6,17 & 2,87 & $-28,73$ & -- & -- & --- \\
\hline Silimarina + alcohol & 4 & 35,74 & 7,86 & 3,66 & $-12,51$ & 1,32 & 0,232 & No sig \\
\hline
\end{tabular}

X TBAR: promedio del resultado de la prueba TBARS DE: desviación estándar
EE: error estándar

Obs: observación
Sig: significativo de leucemia, parásitos, tumores, anemias, insuficiencia cardiaca o enfermedad cardiaca congénita, además de otros trastornos metabólicos ${ }^{(7,15)}$. Este abuso del alcohol altera las células del hígado, que aceleran su apoptosis; aunque el hígado tiene la capacidad de regenerar las zonas afectadas, va dejando lóbulos; por lo que, si el alcoholismo persiste, el tejido del hígado se hará fibroso y las células ya no podrán regenerarse.

El alcohol no solo afecta al hígado, uno de los primeros órganos en ser afectados; Álvarez-Sala y col. señalan que también genera problemas cardiovasculares ${ }^{(38)}$.

En los animales con tratamiento, a las 72 horas y 7 días se observa que la relación peso del hígado/peso del animal se mantuvo siempre incrementado o mayor respecto al grupo que consumió cas-sem en sus alimentos, como se puede apreciar las figuras 1 y 2 . El hígado presentó incremento tamaños (hepatomegalia), hasta $58,69 \%$, a las 72 horas de consumo continuo de alcohol $(p=0,0021)$. Además, podemos señalar que también se observó un cambio de color en el órgano, siendo más intensamente rojizo, a diferencia del color normal, que tiene mas presencia del color marrón.

Berrocal (39) indica que los cambios de coloraciones de los órganos señalan problemas internos del metabolismo, coloraciones que para el caso del hígado se relacionan con enfermedades virales, metabólicas y con el alcoholismo.

Esta diferencia en los pesos de los hígados de los animales tratados con alcohol se debe también al edema y aumento del estrés oxidativo causado por el alcohol.
Tolomeo, citado por Boza López ${ }^{(11)}$, indica que las enfermedades hepáticas son enfermedades relacionadas al estrés oxidativo de las células y que los mecanismos que atenúan estos efectos de los radicales libres son las sustancias antioxidantes, como vitaminas E, A, C, B6, carotenos, flavonoides, selenio, magnesio y zinc, así como compuestos vegetales, como polifenoles, taninos solubles y condensados, flavonoides, lignanos, fenoles sencillos, naftoquinonas; varios de ellos son componentes del cas-sem.

Entre los componentes de las cáscaras y semillas de la uva se encuentra el resveratol, que es un antioxidante natural; según Vázquez, Marcela y col. ${ }^{(8)}$, previene los cambios bioquímicos en ratas, señalando además que en los animales de experimentación se recupera la actividad de las especies del óxido nítrico, potente 


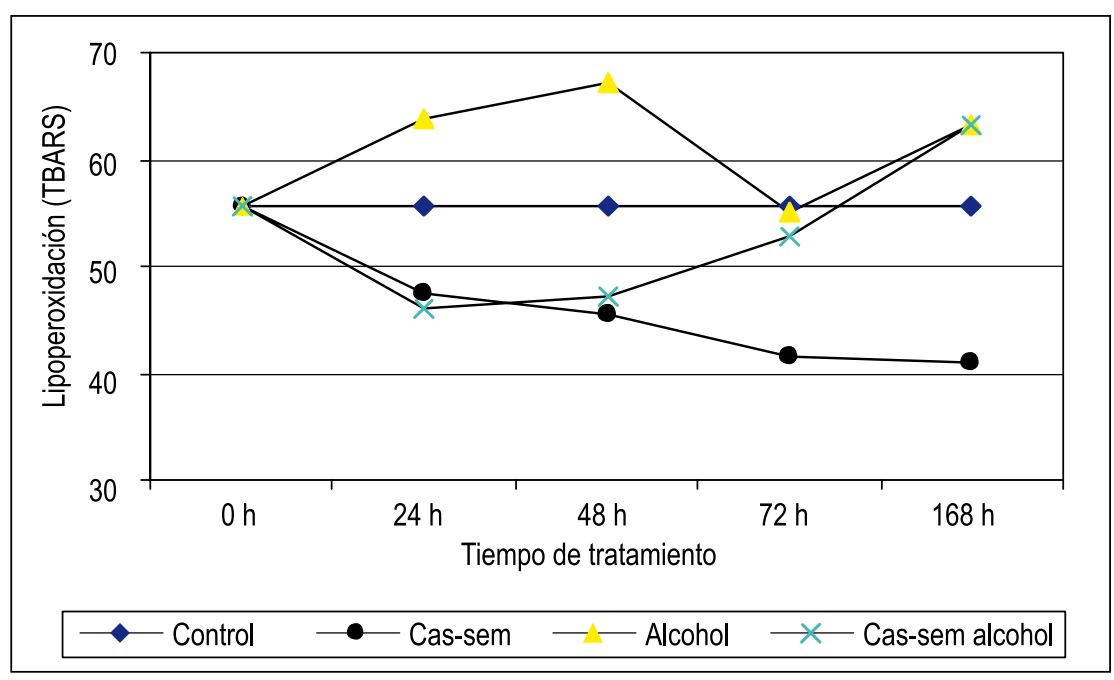

Figura 3. Lipoperoxidación (TBARS) hepática de Mus musculus, según tiempo y tratamiento.

mediador del bienestar cardiovascular, y mejoran los niveles de peroxidación lipídica, tal como nosotros también lo hemos encontrado con la prueba de TBAR, como más adelante detallaremos.

La actividad del cas-sem, de detener o evitar la hepatomegalia en los animales tratados con alcohol, se puede deber a que el preparado del cas-sem al 20\% incluyó cáscaras y semillas de uva, que es donde se localiza la mayor concentración de resvertarol -especialmente en la cáscara-; su extracción se realiza por solubilidad etanólica, que es característica del compuesto, como lo indican Jeandet y col. ${ }^{(37)}$. Así

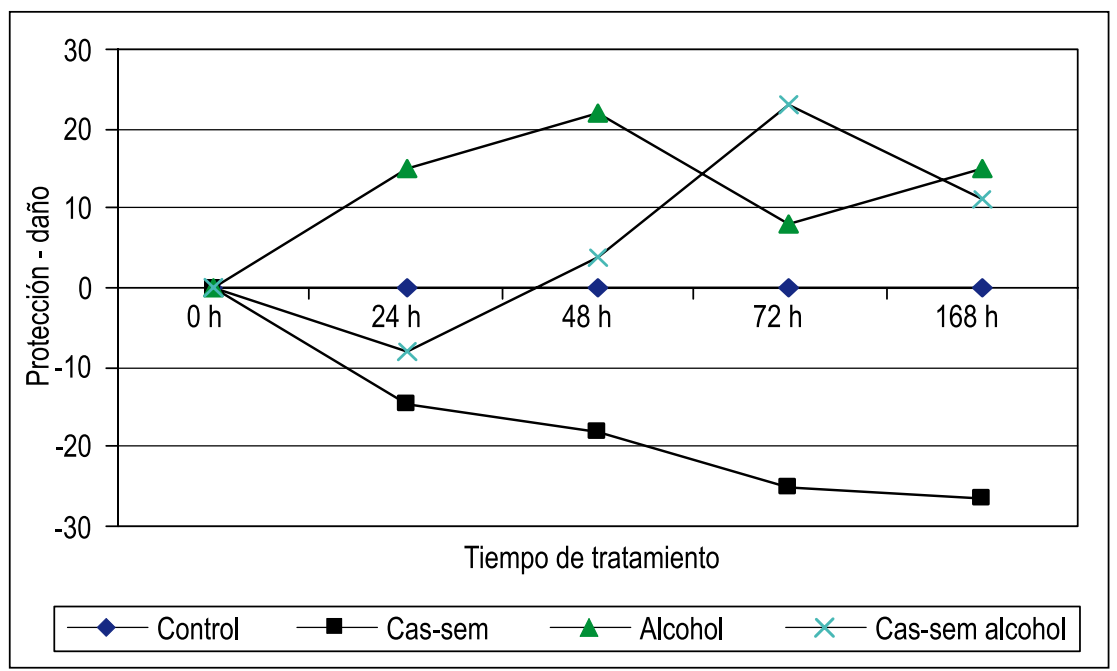

Figura 4. Porcentaje de daño y protección hepática de Mus musculus, según tiempo y tratamiento. to, enzimas, entre otras). El daño puede generarse por la unión misma o por la generación de una respuesta inmunológica (autoanticuerpos) a estos compuestos (aductos), lo que genera inflamación e incremento del volumen y peso del hígado, tal como lo hemos observado en nuestros resultados (ver figuras 3 y 4 ).

Estos productos de degradación producen daño a nivel de membrana, especialmente a los lípidos que la constituyen, observándose la peroxidación de lípidos, en donde se aprecia que el acetaldehido y los radicales libres del oxígeno pueden promover la producción de peróxidos, que atacan los ácidos insaturados y, por consiguiente, la integridad de las membranas, generando entre los productos al malondialdehido. Como señalan Ohkawa y col. ${ }^{\left({ }^{40)}\right.}$ y Lucas y col. ${ }^{(41)}$, el método usado más frecuentemente para evaluar la lipoperoxidación es la medición del malondialdehido, como sustancia reactiva al ácido tiobarbitúroico (TBARS), el cual se fundamenta en la reacción que tiene este ácido con la muestra; adicionándole un cromógeno ${ }^{(42)}$, se puede medir su absorbancia, prueba de TBARS que hemos usado en esta investigación.

La medición de la capacidad antioxidante se realiza de diferentes maneras. Entre estas se encuentra la prueba del TBARS. Esta prueba, también denominada prueba de observación de la lipoperoxidación, mide el daño causado a los lípidos, es decir, el nivel de peroxidación sufrida como consecuencia de la actividad oxidante. El procedimiento que hemos seguido es comparar el resultado de la prueba TBARS en los hígados de los animales con diferente tratamiento y el daño con el control; así mismo, observar la significancia estadística.

Los resultados en la tabla 2 muestran la lipoperoxidación por la prueba TBARS a las 24 horas de tratamiento; el único grupo que sufrió daño fue el que ingirió alcohol, con un incremento del TBARS a $63,91+7,51 \mathrm{nmol} / \mathrm{g}$, lo que corresponde a un daño de $15,11 \%$, a diferencia de los demás grupos, en los que hubo protección, incluso en los animales que consumieron alcohol con cas-sem.

Como ya se ha manifestado, los componentes antiestrés oxidativo de la 
cáscara y semillas de la uva hacen posible esta acción protectora y, más aún, conociendo que los compuestos fenólicos y otros antioxidantes son extraídos por su solubilidad en alcohol, lo que explicaría porqué en el grupo que consumió cassem y alcohol la lipoperoxidación fue menor.

En la tabla 2 se observa los resultados a las 48 horas de tratamiento y se puede apreciar que los animales que consumieron alcohol siempre tienen mayor lipoperoxidación (67,07 nmol/g tejido), que se traduce en un daño de $20,80 \%$, con significancia estadística $(p=0,003)$, mientras los que consumen alcohol con cas-sem siguen siendo protegidos y la variación no es significativa, $47,3 \mathrm{nmol} / \mathrm{g}$ tejido en este grupo, en comparación al grupo cas-sem, con 45,51 nmol/g tejido.

La protección del hígado frente a la agresión del alcohol y sus derivados no es únicamente por el control antiestrés oxidativo, sino que también existe un sistema enzimático de defensa antioxidante, que en combinación con los compuestos flavonoides, fenólicos y otros anti-oxidantes ${ }^{(23,26,32)}$ presente en el cas-sem, permiten esta protección al hígado, como se observa.

Sin embargo, a las 72 horas y después a los 7 días, el panorama cambia. Las defensas antioxidantes provenientes del cas-sem, aparentemente no son suficientes para continuar protegiendo al hígado del ataque agresor del alcohol. Esto lo comentamos en virtud a los resultados observados en la tabla 2, en la que se aprecia que el valor de TBARS en el grupo que consumió cas-sem sigue siendo bajo, 41,55 y 40,85 nmol/g tejido, a las 72 horas y 7 días, respectivamente, mientras que los animales con alcohol evidentemente continuaron con niveles elevados $(52,40$ y $61,91 \mathrm{nmol} / \mathrm{g}$ tejido, a las 72 horas y 7 días, respectivamente), así como en los animales que consumieron alcohol y cas-sem simultáneamente $(52,74$ y $63,1 \mathrm{nmol} / \mathrm{g}$ tejido a 72 horas y 7 días, correspondientemente), como se puede apreciar en las figuras 3 y 4 .

Por lo anteriormente descrito, podemos señalar que efectivamente el cas-sem tiene un efecto hepatoprotector ante la injuria causada por alcohol, protección que es efectiva hasta las 72 horas de ingesta continua del alcohol. Luego, dicha protección desciende al $7^{\circ}$ día de la agresión con alcohol, la cual fue constante en agua del bebedero, a una concentración de $5 \%$ en volúmenes (figura 4). Empero, la protección por consumo de cas-sem al $20 \%$ en la dieta continúa su acción hepatoprotectora, al observar una reducción sostenida del valor de la lipoperoxidación, desde el primer hasta el séptimo día de tratamiento $(47,46 ; 45,51$; 41,55 y $40,85 \mathrm{nmol} / \mathrm{g}$ tejido), como se muestra en la figura 3 .

En un experimento adicional, realizado a un tiempo de consumo de 5 días, se observó que la protección del cas-sem ante la injuria del alcohol fue efectiva hasta los 5 días de tratamiento, encontrando TBARS de 55,88 nmol/g tejido en el grupo alcohol y 44,68 nmol/g tejido en el grupo cas-sem alcohol. En cambio, a los 7 días de tratamiento la TBARS fue de $61,91 \mathrm{nmol} / \mathrm{g}$ tejido en el grupo alcohol y $63,10 \mathrm{nmol} / \mathrm{g}$ tejido en el grupo cas-sem alcohol, entre los cuales ya no hay diferencia significativa.

Referente al tratamiento realizado con silimarina, éste se hizo con el fin de observar y comparar el daño del alcohol frente a un compuesto cuya acción hepatoprotectora está demostrada en diversas investigaciones. Dichos estudios han comprobado que la silimarina presenta un efecto protector frente al tetracloruro de carbono o toxinas, como la faloidina de Amanita phalloides. Este efecto parece ser debido a una alteración de la permeabilidad de membrana, impidiendo el acceso de las toxinas al interior de las células. Por otra parte, la silimarina disminuye la actividad de las células de Kupffer (reduciendo la producción de radicales superóxido, de óxido nítrico y de leucotrienos) y la producción de glutatión, disminuyendo además su oxidación. También, se dice que la silimarina actúa en ensayos in vitro como regenerador hepático. Incrementa la síntesis proteica en el hepatocito, al estimular la actividad de la ARN polimerasa I.

Los animales que fueron tratados con silimarina estuvieron protegidos; así, al observar los resultados se aprecia la protección en los diferentes días de tra- tamiento, evidenciando un descenso de los valores de TBARS, siendo menor que los valores proporcionados por el cas-sem; los valores variaron desde $37,28 \mathrm{nmol} / \mathrm{g}$ tejido hasta $29,11 \mathrm{nmol} / \mathrm{g}$ tejido, llegando a proteger hasta en $47,56 \%$ más, respecto al grupo control.

Para el caso de los ratones que tuvieron en su dieta silimarina y alcohol, también se observó protección, siendo los valores de TBARS mayores que en los animales que consumieron el cas-sem; es decir, estuvieron protegidos, pero el alcohol elevó la lipoperoxidación.

La acción de los flavonoides, compuestos fenólicos, fitatos, vitaminas y otros antioxidantes permitió realizar hepatoprotección. Sin embargo, no es allí únicamente donde actúan. Estudios recientes demuestran que el vino tiene componentes antioxidantes que evitan la oxidación de las LDL-colesterol, favoreciendo la homeostasis del colesterol. Así mismo, en voluntarios se observa que, tras el consumo de vino, aumentó la capacidad antioxidante del plasma.

Otros estudios, como los de Pari ${ }^{(43)}$, señalan la utilidad del extracto de hojas de uva, como una sustancia que reduce significativamente los marcadores de estrés oxidativo en hígado y riñón, tras la administración de alcohol, mejorando la condición antioxidante de los tejidos, resultados comparables a los nuestros, utilizando las cáscaras y semillas de la uva.

En nuestras condiciones experimentales, las cáscaras y semillas de Vitis vinifera L., madura, del Valle de Cañete, desecadas, trituradas (cas-sem) y administradas por vía digestiva en la dieta al 20\% a Mus musculus cepa cb/val453, machos adultos, confiere capacidad hepatoprotectora frente a la injuria provocada por alcohol al $5 \%$, hasta el quinto día.

\section{REFERENCIAS BIBLIOGRÁFICAS}

1. Autónoma Universidad de México. Acción de algunos antiinflamatorios no esteroideos sobre la lipoperoxidación hepática inducida por etanol. Facultad de Medicina, Universidad Nacional Autónoma de México (UNAM), Universidad Autónoma Metropolitana-Xochimilco. Rev Cubana Invest Biomed. 2003;22(1): 16-24.

2. Andriambeloson E, Magnier C, Haan-Archipoff G, Lobstein A, Anton R, Beretz A, et al. Natural dietary 
polyphenolic compounds cause endotheliumdependent vasorelaxation in rat thoracic aorta. J Nutr. 1998;128:2324-33.

3. Aquino A, Astoquilca C, Bayona G. Actividad hepatoportectora del extracto hidroalcohólico de las hojas de Bexa orellana L. en ratas con intoxicación aguda por tetracloruro de carbono. Lima: Universidad Nacional Mayor de San Marcos; 2003.

4. Areguesta-Robles U, Hernández-Ruiz E, Juárez M, Rendón-Ramírez A. Lipoperoxidación y alcoholismo en jóvenes. Bioquimia 2004;29 (Supl 1):125.

5. Buxaderas S, López E, De la Torre M. Actualidades sobre los efectos benéficos del vino en la salud. Simposio Internacional de LASVIN, Junio 2003. Brasil.

6. Ferreira R. Importancia del vino como antioxidante en la prevención de daño oxidativo [monografía en Internet]. Antioxidantes Vitaminas y Nutrientes [revista en Internet]. 2002;(2) [citado el 10 de agosto de 2008]. Disponible en: http://www. antioxidantes.com.ar/Home2.htm

7. Leighton F, Urquiaga I. Polifenoles del vino y salud humana. Antioxidantes y calidad de vida. 2000;7(27):5-13.

8. Vásquez M, Gonzalez S, Risler N, Ponce A, Cruzado M, Miatello R. Un antioxidante natural, resveratrol, previene cambios bioquímicos cardiovasculares en ratas con un modelo de Síndrome X [resumen en Internet]. Mendoza, Argentina: Sociedad Argentina de Cardiología; 2002 [citado el 22 de agosto de 2008]. Disponible en: http://www.sac. org.ar/Sociedades/mesa03.htm.

9. Valls J, Lampreave M, Nadal M, Arola L Importancia de los compuestos fenólicos en la calidad de los vinos tintos de crianza. Tarragona: Universitat Rovira i Virgili; 2000.

10. Zhou HB, Chem JJ, Wang WY, Cai JT, Du Q. Actividad anticáncer del resveratrol en la células gástricas primarias humanas implantadas de carcinoma en ratones desnudos. Mundo J Gastroenterol. 2005;11(2):280-4.

11. Boza J. Alimentación y enfermedad. Anales de la real academia de ciencias veterinarias de Andalucía Oriental. 2003;16(1):163-97.

12. Castillejo N. Nuevos datos sobre los mecanismos de acción del resveratrol [monografía en Internet]. Barcelona, España: noticias.com; 2005 [citado el 24 de junio de 2008]. Disponible en: http://www. noticias.com/notaprensa/08-08-2005/sprimbox/

13. Leighton F, Urquiaga I, Diez M. Propiedades antioxidantes del vino y sus componentes. $77^{\mathrm{a}}$ Asamblea General de la OIV y XXII Congreso de la Vid y del Vino. Buenos Aires, Argentina. 1997.

14. Renaud S, De Lorgeril M. Wine, alcohol, platelets, and the French paradox for coronary heart disease. Lancet. 1992;339:1523-6.

15. Hertog MGL, Feskens EJM, Hollman PCH, Katan MB, Kromhout D. Dietary antioxidant flavonoids and risk of coronary heart disease: the Zutphen Elderly Study. Lancet. 1993;342:1007-11.
16. Hein HO, Suadicani P, Gyntelberg F. Alcohol consumption, serum low density lipoprotein cholesterol concentration, and risk of ischaemic heart disease: six year follow up in the Copenhagen male study. Br Med J. 1996;312:736-41.

17. McElduff P, Dobson AJ. How much alcohol and how often? Population based case-control study of alcohol consumption and risk of a major coronary event. Br Med J. 1997;314:1159-67.

18. Jano $P$, Magnere V. Efectos del riego deficitario en la calidad de mostos y vinos en variedades viníferas finas. Tesis para optar el título profesional de Ingeniero Agrónomo. Facultad de Agronomía e Ingeniería Forestal, Pontificia Universidad Católica de Chile. 2003.

19. Lamuela R. Efectos fisiológicos Cava. Foro Vino ante el siglo XXI, Facultad de Farmacia, Universidad de Barcelona. España. 2002.

20. Minaño A, Chico J, López E, Sisneigas M, Bobadilla M. Efecto de la concentración de sacarosa en la producción de antioxidantes a partir de cultivos celulares de Vitis vinifera L. VAR. Red globe. Rev Peru biol. 2004;11(2):187-92.

21. Bagchi D, Bagchi M, Stohs SJ, Das DK, Ray SD, Kuszynski CA, et al. Free radicals and grape seed proanthocyanidin extract: importance in human health and disease prevention. Toxicology. 2000;148:187-97.

22. Ciudad C, Valenzuela J. Contenido de flavonoides en uvas para vino cultivadas en valle de casablanca, Chile. Agricultura Técnica (Chile). 2002;62(1):7986.

23. Desmarchelier C, Ciccia G. Antioxidantes de origen vegetal. Rev Ciencia Hoy. 1998;8(44):33-8.

24. Sies H. Oxidative stress: from basic research to clinical application. Am J Med. 1991;91:31S39S.

25. Halliwell B. Oxidative stress, nutrition and health. Free Radic Res. 1996;25:57-74.

26. Hernando Y. Polifenoles del vino. La clave de su capacidad antioxidante. Boletín Ciencia, Vino y Salud. 1998;2(2):7-8.

27. Berliner JA, Heinecke JW. The role of oxidized lipoproteins in atherogenesis. Free Radic Biol Med. 1996;20:707-27.

28. Dean RT, Fu S, Stocker R, Davies MJ. Biochemistry and pathology of radical-mediated protein oxidation. Biochem J. 1997;324:1-18.

29. Taylor A. Cataract: relationship between nutrition and oxidation. J Am Coll Nutr. 1993;12:138-46.

30. Gerster H. b-Carotene, vitamin E and vitamin C in different stages of experimental carcinogenesis. Eur J Clin Nutr. 1995;49:155-68.

31. Bravo L. Polyphenols: Chemistry, dietary sources, metabolism, and nutritional significance. Nutr Rev. 1998;56(11):317-33.

32. Leighton F, Urquiaga I, Diez M. Propiedades antioxidantes del vino y sus componentes. $77^{\mathrm{a}}$ Asamblea General de la OIV y XXII congreso de la Vid y del Vino. Buenos Aires, Argentina. 1997.

33. Delmas D, Rebe C, Lacour S, Filomenko R, Athias A, Gambert P, Cherkaoui-Malki M, Jannin B, Dubrez-
Daloz L, Latruffe N, Solary E. Resveratrol-induced apoptosis is associated with Fas redistribution in the rafts and the formation of a death inducing signaling complex in colon cancer cells. J Biol Chem. 2003;278:41482-90.

34. Diplock AT, Charleux JL, Crozier-willi G, Kok FJ, Rice-Evans C, Roberfroid M, et al. Functional food science and defence against reactive oxidative species. Br J Nutr. 1998;80(I):S77-S112.

35. Saldaña-Balmori Y, Ramírez-González B., DelgadilloGutiérrez H. Acción de algunos antiinflamatorios no esteroideos sobre la lipoperoxidación hepática, inducida por etanol. Rev Cubana Invest Biomed 2003:22(1):16-24.

36. Stewart JR, Artime MC, O'Brian CA. Resveratrol: A candidate nutritional substance for prostate cancer prevention. J Nutr. 2003;133:2440S-2443S.

37. Jeandet $P$, Bessis $R$, Gautheron $B$. The production of resveratrol (3,5,4'-trihydroxystilbene) by grape berries in different developmental stages. Am J Enol Vitic. 1991;42:41-6.

38. Álvarez-Sala LA, Valderrama M, Porres A, Gómez Gerique JA, Rodríguez-Gorostiza FI, Torres F, et al. Alcohol y enfermedad cardiovascular. Cardiovascular Risk Factors. 2000;9(5):328-41.

39. Berrocal A, Rodríguez J, Valverde A. Prototecosis sistémica en un canino: descripción patológica de un caso. Arch med vet. 1997;29(2): 307-12.

40. Ohkawa H, Ohishi N, Yagi K. Eassay for lipid peroxides in animal tissues by thiobarbituric acid reaction. Analytical Biochemistry. 1979;95:3518.

41. Masnatta LD, Fischer PA, Domínguez GN, Cabrera El, Ramirez AJ, Sanchez RA. Marcadores de estrés oxidativo. Rev Fed Arg Cardiol. 2003;32:177-83.

42. Soleas G, Goldberg D, Ng E, Karumanchiri A. Tsang $E$, Diamandis E. Comparative evaluation of four methods for assay of cis- and trans- resveratrol. Am J Enol Vitic. 1997;48(2):169-76.

43. Pari L, Suresh A. Effect of grape (Vitis vinifera L.) leaf extract on alcohol induced oxidative stress in rats. Food and chemical toxicology. 2008;46(5):1627-34.

Manuscrito recibido el 12 de octubre de 2008 y aceptado para publicación el 25 de noviembre de 2008.

Correspondencia:

Lic. TM. Miguel Sandoval Vegas

Centro de Investigación de Bioquímica y Nutrición,

Facultad de Medicina, UNMSM.

Av. Grau 755. Lima 1, Perú.

Correo-e: mhsave@gmail.com 